\title{
Controversies in Preconditioning
}

\author{
Jan Willem de Jong, ${ }^{1}$ Robert de Jonge, ${ }^{1}$ \\ Andrea Marchesani, Maarten Janssen, ${ }^{2}$ \\ and Silvia Bradamante ${ }^{2}$ \\ ${ }^{1}$ Cardiochemical Laboratory, Thorax Center, Erasmus University \\ Rotterdam, The Netherlands; ${ }^{2}$ CNR-Centro Sintesi e Stereochimica \\ Speciali Sistemi Organici, Università di Milano, Milan, Italy
}

\begin{abstract}
Summary. Preconditioning is an effective means of protecting the heart against prolonged ischemia by pretreating it with a minor insult, and the present paper reviews various controversies in this highly active field of research. In many models, adenosine plays a role by triggering the activation of protein kinase $C$. It may work in conjunction with other agents, such as bradykinin, but the putative role of noradrenaline is uncertain. Regulation of the enzyme producing adenosine (i.e., 5 '-nucleotidase) has been reported during preconditioning but, because its activity does not seem to be associated with infarct size, it is unlikely that the hydrolase plays a pivotal role. Controversial data have been published on the involvement of mitochondrial ATPase, which may be ascribed to the poor time resolution of the experiments described; however, we do not believe that either acidosis or tissue ATP are important factors in triggering preconditioning. The role of glycolysis in the preconditioning effect remains to be firmly established; opposite mechanisms are activated in low-flow and stop-flow protocols. Although species differences regarding preconditioning exist, they seem to be more of a quantitative than a qualitative nature. The phenomenon could be clinically relevant because evidence is accumulating that preconditioning may take place during bypass surgery and coronary angioplasty if longer balloonocclusion times are used.
\end{abstract}

Cardiovasc Drugs Ther 1996;10:767-773

Key Words. adenosine, angioplasty, ATPase, catecholamines, glycolysis, $5^{\prime}$-nucleotidase, preconditioning, protein kinase $\mathrm{C}$

Ischemic preconditioning makes use of a mild stress in order to activate endogenous defense mechanisms and so enable protection from further major stress. All of the protocols described in the literature lead to a first-time window of protection lasting 60-120 minutes [62]. Originally, Murry et al. [32] used the limitation of infarct size as the criterion establishing the efficacy of an intervention, but subsequent studies have adapted a host of other variables to assess reduction of cell damage, including contractile, morphological, electrophysiological, and biochemical parameters. This article reviews various controversies in this highly active field of research.

\section{Adenosine and Catecholamines}

In ischemic preconditioning, endogenous adenosine protects the heart by stimulating the adenosine $\mathrm{A}_{1^{-}}$ receptor. However, comparison between the cardioprotection induced by exogenous adenosine and that induced by preconditioning shows that discrepancies exist regarding the time frame [61] and extent of protection [55]. Goto et al. [17] proposed that a threshold level of protein kinase C (PKC) stimulation must be reached before cardiac protection by ischemic preconditioning takes place (Fig. 1), and that adenosine and bradykinin released during preconditioning could play major roles in triggering protection by stimulating the kinase, provided that the activity exceeds the threshold [17]. Others have suggested that components such as norepinephrine [50] play a role in reaching the threshold, and Downey's group has reported that hypoxia also preconditions rabbit myocardium via adenosine and catecholamine release [8].

In line with this observation, ischemic preconditioning failed to limit infarct size in a study of rabbit hearts depleted of norepinephrine [49]. Using a similar approach, Weselchouch et al. [58] found no evidence that catecholamines are involved in preconditioning in the rat model. In the same species, $\mathrm{Hu}$ and Nattel [20] reported that stimulation of $\alpha_{1-}$ adrenoceptors causes ischemic preconditioning, but recently Moolman et al. [31] have found no evidence of such a mechanism. In agreement with other observations [44], our own data [12] show that short periods of ischemia, such as those used during preconditioning, do not lead to norepinephrine release into the effluent of isolated rabbit hearts. Furthermore, as reported recently by Seyfarth et al. [45], preconditioning suppresses norepinephrine release after a long

Address for correspondence Dr. Jan Willem de Jong, Cardiochemical Laboratory, Thorax Center, EE 2371, Erasmus University Rotterdam, P.O. Box 1738, 3000 DR Rotterdam, The Netherlands.

Received 15 May 1996; receipt/review time 43 days; accepted in revised form 12 August 1996. 


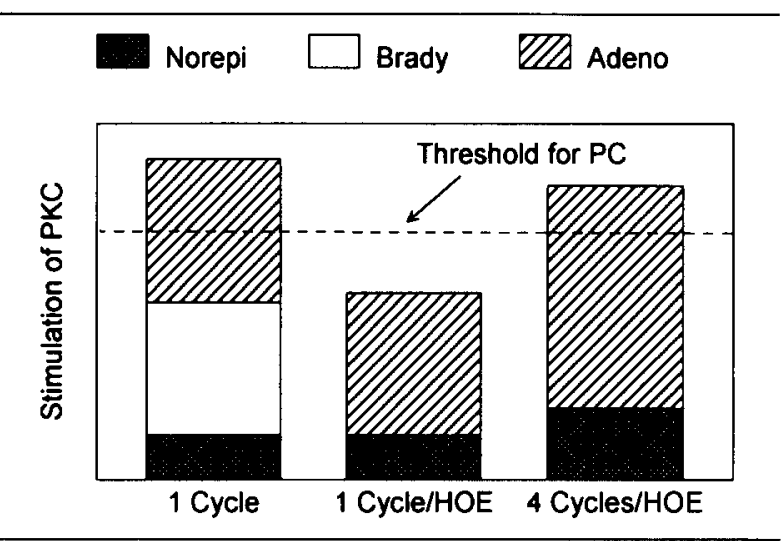

Fig. 1. Hypothetical threshold of protein kinase C (PKC) stimulation that must be reached before cardiac protection takes place by ischemic preconditioning $(P C)$. One cycle of preconditioning produces substantial amounts of adenosine (Adeno) and bradykinin (Brady), in addition to norepinephrine (Norepi), which is enough to simulate $P K C$. If the second component is absent due to bradykinin $B_{2}$-receptor blockade with $H O E 140$, extra cycles are needed to produce enough agonist for PKC stimulation and preconditioning. (After Goto et al. [17], with permission.)

ischemic period. These authors suggested that transient ischemia may have a protective effect on neural tissue by reducing norepinephrine-induced damage during extended periods of myocardial ischemia. We believe that this is a more likely mechanism than protection induced by high catecholamine levels. Taken together, these observations suggest that a combination of agents mediate ischemic preconditioning, and further investigation is clearly needed to evaluate the potential interrelated actions of adenosine norepinephrine.

\section{Hydrolases}

\section{5 '-nucleotidase}

After investigating the relationship between adrenoceptors and preconditioning, Kitakaze's group reported that 5 '-nucleotidase activity increases during $\alpha_{1}$-adrenoceptor stimulation [26]; they also reported increases in $5^{\prime}$-nucleotidase and therefore adenosine production after preconditioning [25]. This latter finding conflicts with results obtained by other groups $[12,39]$. The older methodology used by Kitakaze et al. to measure phosphatase is aspecific and poorly documented [25,26]; although Przyklenk et al.[38] reported an increase in $5^{\prime}$-nucleotidase activity, they did not find any association with infarct size. It is thus unlikely that 5 '-nucleotidase triggers preconditioning in the dog heart.

\section{Mitochondrial ATPase}

Within seconds after the onset of ischemia and the cessation of the electrochemical gradient across the inner mitochondrial membrane, the mitochondrial
ATP-synthase changes into an ATPase $\left(\mathrm{mF}_{1} \mathrm{~F}_{0}\right.$ ATPase). As reviewed by Rouslin [40], inhibition studies with oligomycin show that most of the ATP hydrolyzed during global ischemia is due to $\mathrm{mF}_{1} \mathrm{~F}_{0^{-}}$ ATPase activity. The binding of ATPase inhibitor $\left(\mathrm{IF}_{1}\right)$ to the ATPase leads to reduced ATP hydrolysis. $\mathrm{IF}_{1}$ is an unidirectional inhibitor whose binding is reversible upon the resumption of oxidative metabolism. Protonic inhibition of the complex prevents ATP squandering by $\mathrm{mF}_{1} \mathrm{~F}_{0}$-ATPase activity in ischemic heart cells, probably by means of $\mathrm{IF}_{1}$ binding.

Various groups have examined whether ATPase inhibition may explain the improved energy balance observed in preconditioned hearts [32]. In rat hearts perfused according to Langendorff, preconditioning induced greater ATPase inhibition and less highenergy phosphate depletion during sustained global ischemia [56]. However, Vander Heide et al. [52] concluded that altered $\mathrm{mF}_{1} \mathrm{~F}_{0}-\mathrm{ATPase}$ activity in regionally ischemic dog hearts is not responsible for the energy-sparing effect of ischemic preconditioning. These results are surprising because species with fast heart rates, such as the rat, have less $\mathrm{IF}_{1}$ inhibitor protein than those with slow heart rates, such as the dog [40]. However, we would like to point out that Vander Heide et al. [52] only measured ATPase activity after the last 5-minute reperfusion period of the preconditioning protocol, and so the possibility remains that the inhibition of ATPase in preconditioned hearts became faster or stronger during the sustained ischemic period in their study.

Recent data complicate this issue even further. Kobara et al. [27] observed greater ATPase activity during global ischemia and reperfusion in isolated and preconditioned rat hearts than in controls. Furthermore, the degree of ATP depletion during ischemia was no different between preconditioned and control hearts, whereas ATP levels recovered better during reperfusion in preconditioned hearts. The authors concluded that the preservation of ATPase (and adenine nucleotide translocase) activities may be responsible for the improved restoration of high-energy phosphates.

Although a proper ATP level is crucial for cell homeostasis, it is dissociated from postischemic function [33]. We have no reason to believe that the concentration of ATP is an important factor in eliciting preconditioning.

\section{Glycolysis}

The role of preconditioning in glycolysis/glycogenolysis during ischemia, as well as its relationship to ischemic injury, is controversial. Anaerobic glycolysis supports cell function by means of ATP production, but accumulated glycolytic end products (lactate, sugar and triose phosphates, protons originating from ATP hydrolysis [33]) may determine the extent of 
ischemic damage. Consequently, many studies have attempted to relate the beneficial effects of ischemic preconditioning to cardiac glycogen content or glycolytic rates.

\section{Low-flow ischemia}

Preconditioning before low-flow ischemia increases ischemic glycolytic flux and decreases cardiac injury [23]. These observations agree well with the results of studies showing that enhanced glycolysis and maintained glycolytic ATP production by various means lead to reduced damage and improved function upon reperfusion [42,54]. During low-flow hypoxia in rat heart, glucose prevents the loss of sarcolemmal integrity and contractile function [30].

One may pose the question: Why does anaerobic glycolytic flux protect the myocardium subject to hypoxia/low-flow ischemia? The answer seems to be that the ATP derived from anaerobic glycolysis is preferentially used for membrane-ion pumps and the preservation of cell integrity. During moderate ischemia, anaerobic glycolysis is stimulated (the Pasteur effect), whereas during severe ischemia, this effect is inhibited by the accumulation of lactate and protons, and glycolysis ceases [34]. During low-flow ischemia, lactate and protons are continuously washed out of the ischemic heart, thus preventing their accumulation in tissue and the consequent inhibition of anaerobic glycolysis. Therefore, if the accumulation of the potentially toxic products of glycolysis is prevented by sustaining a moderate flow to the heart, glycolysis can be maintained and glycolytic ATP may preserve cellmembrane integrity and reduce $\mathrm{Ca}^{2+}$ overload during reperfusion.

We believe that the maintenance of glycolysis during low-flow ischemia prevents myocardial injury [34]. So far, only one study [23] has reported that ischemic preconditioning increases glycolytic flux and reduces ischemic cell damage. More research is necessary to clarify the important relationship between preconditioning and glycolysis during low-flow ischemia.

\section{Stop-flow ischemia}

Unlike low-flow ischemia, ischemic preconditioning causes a decrease in glycolytic flux during stop-flow global [41] and regional [32,59] ischemia in both isolated [41] and in vivo [32,59] models. Other strategies aiming at decreased glycolytic flux during regional stop-flow ischemia also induce less ischemic injury [18].

Several groups, including Wolfe et al. [59], assume that the beneficial effects of preconditioning are related to the reduced lactate accumulation and proton production that are the result of lower glycolytic rates, despite reduced ATP production. However, Vander Heide et al. [51] dissociated glycogen depletion and the reduction in lactate accumulation and anaerobic glycolytic flux from ischemic damage. Our own nuclear magnetic resonance (NMR) studies also dem- onstrate cardioprotection by preconditioning in isolated rat hearts without any effects on $\mathrm{pH}$ [6], leading us to believe that acidosis is not a key factor in either functional recovery or ischemic injury [41].

Ischemic preconditioning of the heart reduces its preischemic glycogen content. Wolfe et al. [59] correlated the decrease in infarct size caused by preconditioning with glycogen depletion before sustained ischemia, and with the attenuation of intracellular acidosis during ischemia. A longer period of preconditioning ischemia leads to glycogen depletion and a reduction in infarct size, which are both described by an exponential declining curve [5]. However, the hypothesis that diminished glycogen stores per se limit glycolysis, catabolite accumulation, and cell damage is not supported by other results: Myocardial glycogen loading induced by fasting improves the functional recovery of ischemic rat hearts [43], and in a retrospective study the preischemic glycogen content of rat hearts was correlated with the time of day but not postischemic functional recovery [4]. The question remains as to whether reduced glycolytic flux and accumulated glycolytic products mediate or are secondary to preconditioning, but we do not believe that either lactate or proton accumulation is important in this respect.

\section{Glycolysis and adenosine}

Since adenosine has been implicated in ischemic preconditioning, a number of authors have studied its role on glycolytic flux. Various groups $[22,60]$ have shown that by binding to the $\mathrm{A}_{1}$-receptor, adenosine increases glycolytic flux and induces cardioprotection in isolated rabbit and rat hearts subjected to ischemia and hypoxia, respectively, whereas others have found that it reduces glycolysis and improves functional recovery after ischemia in isolated working rat hearts perfused with glucose and palmitate $[14,15]$. These divergent results could be due to the models studied (low-flow vs. no-flow ischemia), the use of different substrates (glucose vs. glucose/fatty acids), and/or differences between species.

\section{Species Differences}

Although several groups have shown that the rat heart behaves differently from the hearts of other species, the isolated, perfused rat-heart model is quite often used to study preconditioning. It is generally believed that endogenous adenosine does not mediate preconditioning in this species but, as recently pointed out by Headrick [19], the relatively high adenosine levels in rat heart during preconditioning require an increase in (ant)agonist receptor concentration. We refer to the discussion by Przyklenk and Kloner [36] on the species (and model) dependency of the arrhythmic component. The same group [37] could not find any translocation of PKC after ischemic precondi- 
tioning, and this failure to reproduce the original observation in rabbit heart [29] may have been due to species differences.

Questions can be raised about the abundant use of inhibitors and activators (often declared to be specific or selective on rather shaky grounds) to probe the proteins involved. The involvement of $\mathrm{K}_{\mathrm{ATP}}$ channels in the preconditioning of larger animals seems clear but may not be essential in rabbits and rats [for review, see [24]. A pertussis toxin-sensitive G-protein may play a central role in ischemic preconditioning across a broad range of species [20], but major differences may exist in relation to the role of protein kinase $\mathrm{C}$ [16]. The involvement of the inhibitory G-protein in the arrhythmic component of preconditioning is also controversial. Nevertheless, some of these discrepancies may be due to the details of the experimental models studied [20].

\section{Evidence for Preconditioning in Human Heart}

\section{Preinfarction angina}

Ischemic preconditioning clearly exists in many species, and possibly in humans. The phenomenon has been investigated during (retrospective) clinical studies, coronary angioplasty, cardiac surgery, and in vitro. In recent clinical trials, it has been found that preinfarction angina seems to decrease creatinekinase release, in-hospital deaths, arrhythmias, leftventricular function, and infarct size $[3,36]$. Ottani et al. [35] used ventriculographically obtained regional wall motion as a means of indirectly determining infarct size and found that this was less in patients with prodromal angina pectoris occurring at rest 24 hours before the infarct than in patients without previous angina, although the two groups had the same area at risk and in both collaterals were absent. However, as indicated in a recent review by Andreotti et al. [2], controversies still exist that might be explained by the use of thrombolysis, the baseline characteristics of the study population, the presence of multivessel disease, infarcted area, collaterals, and the period(s) of angina before infarction. For instance, studies in the period before the use of thrombolytics have shown that preinfarction angina has negative effects [10], a finding that correlates with the observation that preconditioning exerts an effect only if reperfusion takes place within a certain time frame. Andreotti et al. [2] partly explained these discrepancies by the different definitions of preinfarction angina used. Furthermore, in a prospective study, they [2] observed that the infarct size-limiting effect of preinfarction angina was merely related to faster coronary thrombolysis.

\section{Percutaneous transluminal coronary angioplasty}

Deutsch et al. [13] claim that a 90 -second coronary occlusion induces preconditioning in humans, basing

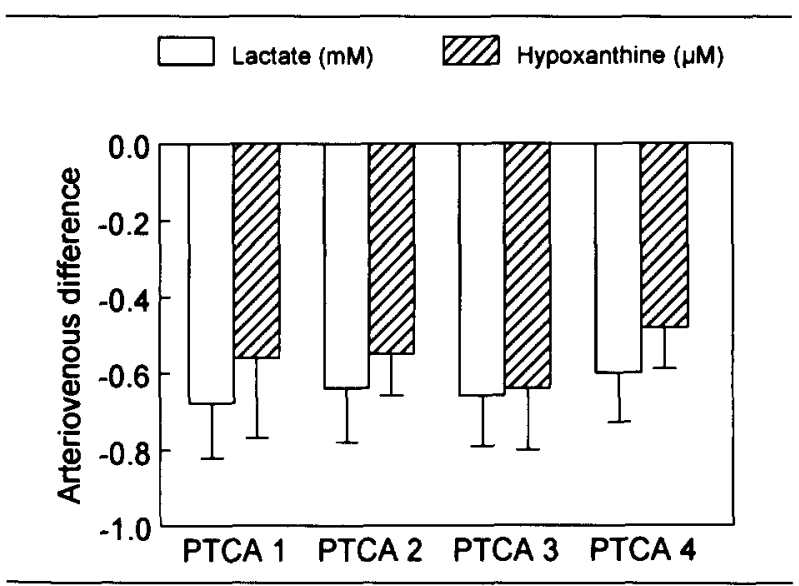

Fig. 2. Lactate and hypoxanthine release after coronary angioplasty. In 28 patients undergoing angioplasty of the left anterior descending coronary artery, arterial and coronary sinus blood samples were taken immediately after occlusions lasting 60-90 seconds. They were rapidly deproteinized with acid and were assayed enzymatically (lactate) or by HPLC (hypoxanthine). Arteriovenous differences are presented means $\pm S E M$.

their conclusions partly on the drop in lactate uptake in 7 out of 12 patients in whom electrophysiological changes during angioplasty were investigated. Jenkins et al. [24] have pointed out that the pretreatment of patients with dipyridamole may potentiate the effect of preconditioning by increasing myocardial adenosine levels.

In a larger patient population $(n=28)$, we measured the myocardial release of lactate and hypoxanthine (an ATP catabolite) immediately after four balloon inflations lasting 60-90 seconds. The arteriovenous difference in both metabolites did not change significantly as a result of the repeated occlusions (Fig. 2). The biochemical markers used provided no evidence for preconditioning during coronary angioplasty [11]. We believe that the angioplasty times of balloon inflations, often lasting less than 2 minutes, are insufficient to induce ischemic preconditioning in humans [24]. In this respect, it is interesting to note that studies using balloon inflations lasting 2 minutes or more $[9,47,48]$ have demonstrated improved tolerance to myocardial ischemia after repetitive coronary occlusions. The exception seems to be a study described later [7]. Further evidence that ischemic preconditioning occurs during angioplasty and is mediated by adenosine $A_{1}$-receptor activation and $\mathrm{K}_{\mathrm{ATP}}$-channel opening has recently been obtained: Adenosine $A_{1}$-receptor blockade by bamiphylline [48] and aminophylline [7], as well as the administration of the selective $\mathrm{K}_{\mathrm{ATP}}$-channel blocker glibenclamide [47], prevent ischemic preconditioning occurring during repetitive balloon occlusions. 


\section{Coronary artery bypass graft surgery}

During both normothermic and hypothermic intermittent aortic crossclamping in coronary artery bypass graft surgery, the release of lactate and inorganic phosphate has been found to be greater during the first than during subsequent reperfusion periods [53]. Yellon's group was the first to report that an ischemic preconditioning protocol slowed the rate of ATP depletion during 10 minutes of crossclamping fibrillation [1], but less ATP depletion in preconditioned hearts gives only indirect evidence of myocardial protection because ischemic cell damage and myocardial function were not reported. It should be noted that the amounts of creatine kinase-MB and lactate released by the ischemic myocardium were no different between the groups, although there was a tendency to lower values in preconditioned hearts. The role of adenosine in ischemic preconditioning has also been studied in the setting of bypass surgery, and it has been found that its presurgery infusion improved intraoperative and postoperative hemodynamic function and reduced ischemic cell damage [28].

\section{Isolated trabeculae and cultured myocytes}

Yellon's group $[46,57]$ exposed human atrial trabeculae to 90 minute of hypoxic substrate-free superfusion, and demonstrated that preconditioning prior to the sustained ischemic period improved functional recovery. This preconditioning effect could be blocked by an adenosine $A_{1}$-antagonist, a PKC antagonist, and a $\mathrm{K}_{\mathrm{ATP}}$-channel blocker. An adenosine $\mathrm{A}_{1}$-agonist, a $\mathrm{K}_{\mathrm{ATP}}$-channel opener, and a $\mathrm{PKC}$ activator mimicked the effect of ischemic preconditioning, whereas the effects of the $\mathrm{K}_{\mathrm{ATP}}$-channel opener and the PKC activator could be blocked by the $\mathrm{K}_{\mathrm{ATP}}$-channel blocker glibenclamide. Ikonomidis et al. [21] demonstrated that the infarct size-limiting effect of ischemic preconditioning also exists in human cultured ventricular cardiomyocytes.

\section{Discussion}

A number of endogenous compounds are candidate triggers of preconditioning: These are adenosine, bradykinin, and noradrenaline, which may work in conjunction to reach a threshold. Although we doubt the importance of catecholamines in this respect, we would like to point out that all of these metabolites activate intracellular signaling pathways via receptors linked to GTP-binding proteins and phospholipases; protein kinase(s) could be stimulated, and thus lead to the phosphorylation of putative target proteins [17]. As reviewed elsewhere in this issue [16], these include regulatory proteins of the $\mathrm{K}_{\mathrm{ATP}}$ channels and the expression of heat-shock proteins (for the second time window of protection), although, as outlined earlier, we do not believe that $5^{\prime}$-nucleotidase belongs to this class of possible targets. However, we do con- sider the possibility that the adenosine released during short periods of ischemia and reperfusion activates PKC, which in turn phosphorylates a glycolytic key enzyme and thus increases glycolytic flux $[23,60]$. The present data do not allow any firm statement to be made regarding the preconditioning role of (the translocation of) PKC in all species. Although there is growing evidence that the human heart can be preconditioned, there is still a lack of crucial information on the subcellular pathways involved (e.g., with PKC).

\section{Acknowledgments}

We gratefully acknowledge the support of the Netherlands Heart Foundation, the European Community (Biomed II), and NATO (project nos. NHS 94.043, N BMH4-CT 950838 , and CRG 920235, respectively).

\section{References}

1. Alkhulaifi AM, Yellon DM, Pugsley WB. Preconditioning the human heart during aorto-coronary bypass surgery. Eur J Cardiothorac Surg 1994;8:270-276.

2. Andreotti F, Pasceri V, Hackett DR, Davies GJ, Haider AW, Maseri A. Preinfarction angina as a predictor of more rapid coronary thrombolysis in patients with acute myocardial infarction. N Engl J Med 1996;334:7-12.

3. Anzai T, Yoshikawa T, Asakura Y, et al. Preinfarction angina as a major predictor of left ventricular function and long-term prognosis after a first $Q$ wave myocardial infarction. J Am Coll Cardiol 1995;26:319-327.

4. Asimakis GK. Myocardial glycogen depletion cannot explain the cardioprotective effects of ischemic preconditioning in the rat heart. J Mol Cell Cardiol 1996;28:563-570.

5. Barbosa V, Sievers RE, Zaugg CE, Wolfe CL. Preconditioning ischemia time determines the degree of glycogen depletion and infaret size reduction in rat hearts. Am Heart $J$ 1996;131:224-330.

6. Bradamante S, Piccinini F, Delu C, Janssen M, De Jong JW. NMR evaluation of changes in myocardial high energy metabolism produced by repeated short periods of ischemia. Biochim Biophys Acta 1995;1243:1-8.

7. Claeys MJ, Vrints CJ, Bosmans JM, Conraads VM, Snoeck JP. Aminophylline inhibits adaptation to ischaemia during angioplasty. Role of adenosine in ischaemic preconditioning. Eur Heart $J$ 1996;17:539-544.

8. Cohen MV, Walsh RS, Goto M, Downey JM. Hypoxia preconditions rabbit myocardium via adenosine and catecholamine release. J Mol Cell Cardiol 1995;27:1527-1534.

9. Cribier A, Korsatz L, Koning R, et al. Improved myocardial ischemic response and enhanced collateral circulation with long repetitive coronary occlusion during angioplasty: A prospective study. J Am Coll Cardiol 1992;20:578-586.

10. Cupples LA, Gagnon DR, Wong ND, Ostfeld AM, Kannel WB. Preexisting cardiovascular conditions and long-term prognosis after initial myocardial infarction: The Framingham Study. Am Heart J 1993;125:863-872.

11. De Jong JW, Cargnoni A, Huizer T, Ferrari R, Bonnier JJRM. Lactate and hypoxanthine release after coronary angioplasty: No evidence of preconditioning (abstr). $J \mathrm{Mol}$ Cell Cardiol 1993;25(Suppl 1):S37. 
12. De Jong JW, Cargnoni A, Bradamante S, et al. Intermittent $\mathrm{v}$ continuous ischemia decelerates adenylate breakdown and prevents norepinephrine release in reperfused rabbit heart. $J$ Mol Cell Cardiol 1995;27:659-671.

13. Deutsch E, Berger M, Kussmaul WG, Hirshfeld JW Jr, Hermann HC, Laskey WK. Adaptation to ischemia during percutaneous transluminal coronary angioplasty. Clinical, hemodynamic, and metabolic features. Circulation 1990;82: 2044-2051.

14. Finegan BA, Lopaschuk GD, Coulson CS, Clanachan AS. Adenosine alters glucose use during ischemia and reperfusion in isolated rat hearts. Circulation 1993;87:900-908.

15. Fralix TA, Murphy E, London RE, Steenbergen C. Protective effects of adenosine in the perfused rat heart: Changes in metabolism and intracellular ion homeostasis. $A m J$ Physiol 1993;264:C986-994.

16. Gho BCG, Eskildsen-Helmond YEG, De Zeeuw S, Lamers JMJ, Verdouw PD. Does protein kinase $C$ play a pivotal role in the mechanisms of ischemic preconditioning? Cardiovasc Drugs Ther 1996;10:775-786.

17. Goto M, Liu Y, Yang X-M, Ardell JL, Cohen MV, Downey JM. Role of bradykinin in protection of ischemic preconditioning in rabbit hearts. Circ Res 1995;77:611-621.

18. Goto M, Tsuchida A, Liu Y, Cohen MV, Downey JM. Transient inhibition of glucose uptake mimics ischemic preconditioning by salvaging ischemic myocardium in the rabbit heart. J Mol Cell Cardiol 1995;27:1883-1894.

19. Headrick JP. Ischemic preconditioning: Bioenergetic and metabolic changes and the role of endogenous adenosine. $J$ Mol Cell Cardiol 1996;28:1227-1240.

20. $\mathrm{Hu} \mathrm{K}$, Nattel $\mathrm{S}$. Mechanisms of ischemic preconditioning in rat hearts. Involvement of $\alpha_{1 \mathrm{~B}}$-adrenoceptors, pertussis toxin-sensitive G proteins, and protein kinase C. Circulation 1995;92:2259-2265.

21. Ikonomidis JS, Tumiati LC, Weisel RD, Mickle DAG, Li $\mathrm{R}-\mathrm{K}$. Preconditioning human ventricular cardiomyocytes with brief periods of simulated ischaemia. Cardiovasc Res 1994;28:1285-1291.

22. Janier MF, Vanoverschelde J-LJ, Bergmann SR. Adenosine protects ischemic and reperfused myocardium by receptor-mediated mechanisms. Am $J$ Physiol 1993;264: H163-170.

23. Janier MF, Vanoverschelde J-LJ, Bergmann SR. Ischemic preconditioning stimulates anaerobic glycolysis in the isolated rabbit heart. Am J Physiol 1994;267:H1353-1360.

24. Jenkins DP, Steare SE, Yellon DM. Preconditioning the human myocardium: Recent advances and aspirations for the development of a new means of cardioprotection in clinical practice. Cardiovasc Drugs Ther 1995;9:739-747.

25. Kitakaze M, Hori M, Takashima S, Sato H, Inoue M, Kamada $\mathrm{T}$. Ischemic preconditioning increases adenosine release and $5^{\prime}$-nucleotidase activity during myocardial ischemia and reperfusion in dogs. Implications for myocardial salvage. Circulation 1993;87:208-215.

26. Kitakaze M, Hori M, Morioka T, et al. $\alpha_{1}$-Adrenoceptor activation increases ecto-5'-nucleotidase activity and adenosine release in rat cardiomyocytes by activating protein kinase C. Circulation 1995;91:2226-2234.

27. Kobara M, Tatsumi T, Matoba S, et al. Effect of ischemic preconditioning on mitochondrial oxidative phosphorylation and high energy phosphates in rat hearts. $J$ Mol Cell Cardiol 1996;28:417-428.

28. Lee HT, LaFaro RJ, Reed GE. Pretreatment of human myocardium with adenosine during open heart surgery. $J$ Card Surg 1995;10:665-676.
29. Liu Y, Ytrehus K, Downey JM. Evidence that translocation of protein kinase $\mathrm{C}$ is a key event during ischemic preconditioning of rabbit myocardium. J Mol Cell Cardiol 1994;26: 661-668.

30. Lochner A, Pentz A, Williams K, Tromp E, Harper IS. Substrate effects on sarcolemmal permeability in the normoxic and hypoxic perfused rat heart. Basic Res Cardiol 1996;91:64-78.

31. Moolman JA, Genade S, Tromp E, Lochner A. No evidence for mediation of ischemic preconditioning by alpha ${ }_{1}$ adrenergic signal transduction pathway or protein kinase $\mathrm{C}$ in the isolated rat heart. Cardiovasc Drugs Ther 1996;10: 125-136.

32. Murry CE, Richard VJ, Reimer KA, Jennings RB. Ischemic preconditioning slows energy metabolism and delays ultrastructural damage during a sustained ischemic episode. Circ Res 1990;66:913-931.

33. Neely JR, Grotyohann LW. Role of glycolytic products in damage to ischemic myocardium. Dissociation of adenosine triphosphate levels and recovery of function of reperfused ischemic hearts. Circ Res 1984;55:816-824.

34. Opie LH. The mechanism of myocyte death in ischaemia. Eur Heart $J$ 1993;14(Suppl G):31-33.

35. Ottani F, Galvani M, Ferrini D, et al. Prodromal angina limits infarct size. A role for ischemic preconditioning. Circulation 1995;91:291-297.

36. Przyklenk K, Kloner RA. Preconditioning: A balanced perspective. Br Heart $J$ 1995;74:575-577.

37. Przyklenk K, Sussman MA, Simkhovich BZ, Kloner RA. Does ischemic preconditioning trigger translocation of protein kinase $\mathrm{C}$ in the canine model? Circulation 1995;92: 1546-1557.

38. Przyklenk K, Zhao L, Kloner RA, Elliott GT. Reduction of infarct size with ischemic preconditioning and monophosphoryl lipid A: Role of adenosine regulating enzymes? Am $J$ Physiol 1996, in press.

39. Reimer KA, Murry CE, Yamasawa I, Hill ML, Jennings RB. Four brief periods of myocardial ischemia cause no cumulative ATP loss or necrosis. Am J Physiol 1986;251: H1306-1315.

40. Rouslin W. Regulation of the mitochondrial ATPase in situ in cardiac muscle: Role of the inhibitor subunit. $J$ Bioenerg Biomembr 1991;23:873-888.

41. Schaefer S, Carr LJ, Prussel E, Ramasamy R. Effects of glycogen depletion on ischemic injury in isolated rat hearts: Insights into preconditioning. Am $J$ Physiol 1995;268: H935-944.

42. Schaefer S, Prussel E, Carr LJ. Requirement of glycolytic substrate for metabolic recovery during moderate low flow ischemia. J Mol Cell Cardiol 1995;27:2167-2176.

43. Schneider CA, Taegtmeyer H. Fasting in vivo delays myocardial cell damage after brief periods of ischemia in the isolated working rat heart. Circ Res 1991;68:10451050.

44. Schömig A, Dart AM, Dietz R, Mayer E, Kübler W. Release of endogenous catecholamines in the ischemic myocardium of the rat. Part A: Locally medicated release. Circ Res 1984;55:689-701.

45. Seyfarth M, Richardt G, Mizsnyak A, Kurz T, Schömig A. Transient ischemia reduces norepinephrine release during sustained ischemia. Neural preconditioning in isolated rat heart. Circ Res 1996;78:573-580.

46. Speechly-Dick ME, Grover GJ, Yellon DM. Does ischemic preconditioning in the human involve protein kinase $\mathrm{C}$ and the ATP-dependent $\mathrm{K}^{+}$channel? Studies of contractile func- 
tion after simulated ischemia in an atrial in vitro model. Circ Res 1995;77:1030-1035.

47. Tomai F, Crea F, Gaspardone A, et al. Ischemic preconditioning during coronary angioplasty is prevented by glibenclamide, a selective ATP-sensitive $\mathrm{K}^{+}$channel blocker. Circulation 1994;90:700-705.

48. Tomai F, Crea F, Gaspardone A, et al. Effect of $A_{1}$ adenosine receptor blockade by bamiphylline on ischaemic preconditioning during coronary angioplasty. Eur Heart $J$ 1996; 17:846-853.

49. Toombs CF, Wiltse AL, Shebuski RJ. Ischemic preconditioning fails to limit infarct size in reserpinized rabbit myocardium. Implication of norepinephrine release in the preconditioning effect. Circulation 1993;88:2351-2358.

50. Vander Heide RS, Schwartz LM, Jennings RB, Reimer KA. Effect of catecholamine depletion on myocardial infarct size in dogs: Role of catecholamines in ischemic preconditioning. Cardiovasc Res 1995;30:656-662.

51. Vander Heide RS, Delyani JA, Jennings RB, Reimer KA, Steenbergen $C$. Reducing lactate accumulation does not attenuate lethal ischemic injury in isolated perfused rat hearts. Am J Physiol 1996;270:H38-44.

52. Vander Heide RS, Hill ML, Reimer KA, Jennings RB. Effect of reversible ischemia on the activity of the mitochondrial ATPase: Relationship to ischemic preconditioning. $J$ Mol Cell Cardiol 1996;28:103-112.

53. Van der Vusse GJ, Van der Veen FH, Flameng W, et al. A biochemical and ultrastructural study on myocardial changes during aorto-coronary bypass surgery: St. Thomas Hospital cardioplegia versus intermittent aortic crossclamping at 34 and $25^{\circ} \mathrm{C}$. Eur Surg Res 1986;18:1-11.

54. Vanoverschelde J-LJ, Janier MF, Bakke JE, Marshall DR, Bergmann SR. Rate of glycolysis during ischemia determines extent of ischemic injury and functional recov- ery after reperfusion. Am $J$ Physiol 1994;267:H17851794.

55. Van Winkle DM, Chien GL, Wolff RA, Soifer BE, Kuzume $\mathrm{K}$, Davis RF. Cardioprotection provided by adenosine receptor activation is abolished by blockade of the $\mathrm{K}_{\mathrm{ATP}}$ channel. Am J Physiol 1994;266:H829-839.

56. Vuorinen K, Ylitalo K, Peuhkurinen K, Raatikainen P, AlaRämi A, Hassinen IE. Mechanisms of ischemic preconditioning in rat myocardium. Roles of adenosine, cellular energy state, and mitochondrial $\mathrm{F}_{1} \mathrm{~F}_{0}$-ATPase. Circulation 1995;91:2810-2818.

57. Walker DM, Walker JM, Pugsley WB, Pattison CW, Yellon DM. Preconditioning in isolated superperfused human muscle. J Mol Cell Cardiol 1995;27:1349-1357.

58. Weselchouch EO, Baird AJ, Sleph PG, Dzwonczyk S, Murray HN, Grover GJ. Endogenous catecholamines are not necessary for ischaemic preconditioning in the isolated perfused rat heart. Cardiovasc Res 1995;29:126-132.

59. Wolfe CL, Sievers RE, Visseren FLJ, Donnelly TJ. Loss of myocardial protection after preconditioning correlates with the time course of glycogen recovery within the preconditioned segment. Circulation 1993;87:881-892.

60. Wyatt DA, Edmunds MC, Rubio R, Berne RM, Lasley RD, Mentzer RM Jr. Adenosine stimulates glycolytic flux in isolated perfused rat hearts by $\mathrm{A}_{1}$-adenosine receptors. $A m J$ Physiol 1989;257:H1952-1957.

61. Yao Z, Gross GJ. A comparison of adenosine-induced cardioprotection and ischemic preconditioning in dogs. Efficacy, time course, and role of $\mathrm{K}_{\mathrm{ATP}}$ channels. Circulation 1994;89:1229-1236.

62. Yellon DM, Baxter GF. A "second window of protection" or delayed preconditioning phenomenon: Future horizons for myocardial protection? J Mol Cell Cardiol 1995;27: 1023-1034. 